\title{
Editorial
}

Alfred W.L. Jay PH D, C.H. Kehler MD FRCPC

\section{Heart perforation by central venous catheters}

Since their introduction in the early 1950s, central venous catheters have become an indispensable tool in the operating room and in the intensive care unit. At least a dozen manufacturers currently sell these devices in North America, and more than 50,000 catheters are used in Canada each year. In a large metropolitan general hospital, as many as 1,000 catheters may be used in patients annually, with the anaesthetist being the major user.

Perforation of the heart and the vena cava by central venous catheters inserted for central venous pressure (CVP) monitoring and for total parenteral nutritional (TPN) therapy have been reported. ${ }^{1-4}$ In the past year, seven cases of perforation resulting in death were reported to the Health Protection Branch, Health and Welfare Canada ${ }^{5.6}$ Six different brands of catheters were involved in these cases which occured in six Canadian hospitals. The frequency of these reported incidents appears to agree with the reported frequency of occurrence of about two cases in a thousand applications. ${ }^{7}$

Softer materials can reduce catheter stiffness ${ }^{8}$ and therefore the potential for perforation. However, incidents involving catheters made of polyvinylchloride (PVC), polyethylene, polyurethane, and teflon have all been reported. ${ }^{4.9}$ Silastic rubber is currently the softest material used for manufacturing catheters. Unfortunately, the addied softness also makes the catheters unsatisfactory for monitoring of pressure-pulse profile, due to damping effects. For TPN and for monitoring of mean venous pressures, they may be safer to use, although tissue erosion, as a result of prolonged tissue contact and vessel wall exposure to hyperosmotic fluids may still result in

From the Bureau of Radiation \& Medical Devices, Health Protection Branch and the Division of Cardiothoracic Surgery, Ottawa Civic Hospital, Ottawa, Ontario (AWLJ), and the Deparment of Anesthesia, University of Manitoba, Winnipeg, Manitoba (CHK).

Address correspondence to: Dr. Alfred Jay, Bureau of

Radiation \& Medical Devices, Health Protection Branch, Tunney's Pasture, Ottawa, Ontario, K1A 0L2. perforation and cardiac tamponade. ${ }^{10}$ The diameter of the catheter, as well as the geometry of its lumen, affects catheter rigidity and are therefore relevant parameters to consider when selecting a catheter for a particular patient or application.

Perforation may occur during insertion, either by a stiff catheter, or by the wire-guide, or it may occur subsequently by an in-dwelling catheter. Perforations during insertion mostly involve perforation of the vessel. and usually can be corrected at the time, without serious consequence, although the damaged tissue may make further advancement of the catheter or the wire-guide even more difficult. Under these circumstances, perforation can be diagnosed if back-flow of blood cannot be demonstrated, such as when the infusion bag is lowered to confurm placement of the catheter tip. Delayed perforations generally result from arm, body, or head movements, depending on the insertion site. For a catheter inserted via a jugular vein, head movements, such as tipping the head, can advance the catheter tip by several centimeters towards the heart. For a catheter inserted through a vein in the arm, extreme arm movements can advance the catheter tip by $6-10 \mathrm{~cm} .{ }^{\text {" Perforation can }}$ also result from the contractile motion of the heart, if the catheter tip is located in the right atrium or right ventricle. Two-thirds of the reported perforations have occurred within the first two days of insertion. ${ }^{5}$ In most cases, perforation resulted in TPN fluid being infused into the pericardial cavity, and in some cases, blood also leaked into the pericardial sac through the perforation site.

The potential for heart and vessel perforation can be reduced by improvements in materials and catheter tip design. Meanwhile, patient morbidity and mortality due to cardiac perforation can be minimized through careful application and increased vigilance. The following are recommended:

- The choice of catheter must take into account the size of the patient, so as to determine the proper length to use.

- In a blood vessel, the tip of the catheter is best positioned where it will be parallel with the vessel walls, avoiding sharp contact with the vessel wall at bifurcations and bends. 
- For TPN and CVP, the tip of the catheter should not be advanced beyond the entrance to the heart, i.e., the junction between the vena cava and the right atrium.

- When the tip of the catheter is specifically placed into the heart, such as for ventricular pressure measurements, the increased potential for heart perforation, and therefore the need for increased vigilance, must be recognized.

- The position of the catheter tip should be confirmed, either by fluoroscopy during insertion, or by $x$-ray immediately after.

- Since movement can advance the tip of the catheter, periodic re-confirmation of tip position is recommended.

- Patients with in-dwelling catheters should be cautioned to limit movements of the upper body, and should not be mobile without appropriate supervision.

- When cardiovascular deterioration occurs in a patient with an in-dwelling central venous catheter, the possibility of catheter perforation and cardiac tamponade should be considered as part of the differential diagnosis.

\section{Acknowledgement}

The authors sincerely appreciate the assistance of Suzette Wadhwani in the preparation of this nanuscript.

\section{La perforation cardia- que causée par des cathéters veineux centraux}

Depuis leur introduction au début des années 1950 , les cathêters veineux centraux sont devenus un outil indispensable en salle d'opération et dans l'unité des soins intensifs. En Amérique du Nord, au moins une douzaine de manufacturiers vendent présentement ces cathéters, et on en utilise au moins 50,000 par année au Canada. Dans l'hôpital général d'une grande métropole il peut arriver qu'on utilise, chez des patients, au moins 1,000 cathéters par année, l'utilisateur principal étant l'anesthésiste.

On a rapporté que des cathéters veineux centraux, installés pour un monitorage de la pression veineuse centrale (PVC) et pour une thérapie de nutrition parentérale totale (NPT), avaient perforé le coeur et la veine cave ${ }^{1,4}$ Dans la dernière année, sept cas de perforation résultant en décès ont été signalés au Direction générale de la Protection de la santé de Santé et Bien-Etre Social Canada. ${ }^{5,6}$ Pour ces cas qui se sont produits dans six höpitaux canadiens, on avait utilisé six marques différentes de cathéters. La fréquence de ces incidences semble concorder avec l'incidence d'environ deux cas par 1000 utilisations. ?

Des matériaux plus souples peuvent réduire la rigidité du cathéter ${ }^{8}$ et par le fait même téduire le potentiel de perforation. Cependant, on a rapporté des incidents où les cathéters faits de chlorure de polyvinyle (CPV), de polyéthylène, de polyuréthane et de teflon étaient utilisés. ${ }^{4,9} \mathrm{Le}$ caoutchouc silastique est présentement le matériau le plus souple utilisé dans la fabrication des cathéters. Malheureusement à cause de l'effet amortisseur qui accompagne cet ajout de souplesse, les cathêters deviennent médiocres pour le monitorage du profil pression-pouls. Leur utilisation peut être plus sécuritaire dans la NPT et dans le monitorage des pressions veineuses moyenries, quiqu'une perforation et une tamponnade cardiaque peuvent encore se produire à la suite d'une érosion tissulaire résultant d'un contact tissulaire prolongé et de l'exposition de la paroi vasculaire aux liquides hyperosmotiques. ${ }^{10}$ Le diamètre du cathêter et la géométrie de sa lumière agissent autant, $l^{\prime}$ un et $l^{\prime}$ autre, sur la rigidité du cathéter; ils sont done l'un et l'autre des paramètres pertinents qui doivent être pris en considération quand on choisit un cathéter pour un patient où pour une utilisation particulière.

La perforation peut se produire lors de l'insertion d'un catheter rigide ou d'un guide métallique, ou elle peut être causée subséyuemment par un cathéter à demeure. La plupart des perforations qui se produisent lors de l'insertion sont des perforations vasculaires, et elles peuvent être habituellement corrigées au moment même sans qu'il n'y ait de conséquences sérieuses, quoique l'avancement plus poussée du cathéter ou du gujde métallique devient plus difficile à cause des tissus endommagés. Dans ces circonstances, on peut diagnostiquer une perforation si le contre-courant sanguin ne peut être démontré, tel que cela se produit quand le sac d'infusion est abaissé afin de s'assurer de l'emplacement du bout du cathéter. Les perforations tardives résultent généralement des mouvements du bras, du corps, ou de la tête selon le site d'insertion. Pour ce qui est d'un cathéter inséré dans une veine jugulaire, les mouvements de tête telle l'inclinaison, peuvent faire avancer le bout du cathéter de plusieurs centimètres en direction du cour. Pour ce qui est d'un cathéter inséré dans une veine du bras, des mouvements extrêmes du bras peuvent faire avancer le bout du cathéter de $6-10 \mathrm{~cm} .{ }^{11}$ La perforation peut aussi être causée par le mouvement contractile cardiaque si le bout du cathéter se situe dans l'oreillette droite ou le ventricule droit. Deux-tiers des perforations rapportées se sont produites 
dans les deux premiers jours de l'insertion. ${ }^{5}$ Dans la plupart des cas le liquide de NPT śtait infusé dans la cavité péricardique suite à la perforation. Dans certains cas, il y avait un écoulement sanguin qui se produisait dans le péricardc par le site de perforation.

Le potentiel de perforation cardiaque et vasculaire peut être réduit en apportant des améliorations aux matériaux et à la forme de l'extrémité du cathéter. Entre-temps, la perforation cardiaque, causant une morbidité et une mortalité chez des patients peut être minimisée par une utilisation attentive et une vigilance accrue. Nous recommandons ce qui suit:

- Dans le choix du cathéter, on doit prendre en considération la taille du patient afin de déterminer la longueur devant être utilisée.

- La meilleure position du cathéter dans un vaisseau sanguin est une position parallèle à celui-ci afin d'éviter un contact aigu avec la paroi vasculaire dans les bifurcations et les courbures.

- Pour la NPT et la PVC, l'extrémité du cathéter ne devrait pas aller au delà de l'entrée du cour, c'est-àdire la jonction entre la veine cave et l'oreillette droite.

- Quand l'extrémité du cathéter est placée de façon spécifique dan le cceur, comme c'est le cas pour les mesures de pression ventriculaires, on doit reconnaître le potentiel accru de perforation cardiaque, et done, la nécessitê đ'une vigilance accrue.

- On devrait confirmer la position de l'extrémité du cathéter par une fluoroscopie durant l'insertion ou par une radiographie prise immédiatement après.

- Puisque le mouvement peut faire avancer l'extrémité du cathéter, on recommande une reconfirmation périodique de la position de l'extrémité. Les patients ayant un cathéter à demeure devraient être avertis de limiter le mouvement de la partie supérieure du corps, et ils ne devraient pas être ambulants sans surveillance appropriée

- Quand une détérioration cardio-vasculaire se produit chez un patient porteur d'un cathếter veineux central à demeure, on devrait considerer que la possibilité de perforation par le cathéter et celle d'une tamponnade cardiaque font partie du diagnostic différentiel.

\section{Remerciements}

Les auteurs apprécient sincèrement l'aide de Suzette Wadhwani dans la préparation de ce manuscrit.

\section{References}

1 Collier PE, Ryan JJ, Diamond DL. Cardiac tamponade from central venous catheters. Report of a case and review of the English literature. Angiology 1984; 35: 595.

2 Eide J, Odegaard E. Cardiac tamponade as a result of infusion therapy. A potentially amenable complication of central venous catheters. Acta Anaesthesiol Scand 1983; 27: 181 .

3 Jay AWL, Aldridge HE. Perforation of the heart or vena cava by central venous catheters inserted for monitoring or infusion therapy. Can Med Assoc J 1986; 135: 1143.

4 Karnauchow PN. Cardiac tamponade from central venous catheterization. Can Med Assoc J 1986; 135: 1145.

5 Aldridge $H E$, Jay AWL. Editorial: Central venous catheters and heart perforation. Can Med Assoc J 1986; 135: 1082.

6 Perforation of the heart and vessel by central yenous catheters inserted for CVP monitoring and TPN therapy. Medical Devices Alert 79, Health Protection Branch, Dept of National Health \& Welfare, Ottawa, March 7, 1986.

7 Burri C, Ahnefeld FW. Caval Catheters, Springer-Verlag, Bcrlin, 1978.

8 Stenquist O, Curelaru I, Linder LE, Gustavsson B. Stiffness of central venous catheters. Acta Anaesthesiol Scand 1983; 27: 153.

9 Greenall MJ, Blewith RW, McMahon MJ. Cardiac tamponade and central venous catheters. Br Med J 1975;2: 595.

10 Harford IF Jr, Kleinsasser J. Fatal cardiac tamponade in a patient receiving total parenteral nutrition via a Silastic central venous catheter. JPEN 1984; 8: 443

11 Krog $M$, Berggren $L$, Brodin $M$, Wickbom $G$. Pericardial tamponade caused by central venous catheters. World J Surg 1982; 6: 138. 